\title{
Catridecacog: a breakthrough in the treatment of congenital factor XIII A-subunit deficiency?
}

This article was published in the following Dove Press journal:

Journal of Blood Medicine

9 July 2014

Number of times this article has been viewed

\section{Wolfgang Korte ${ }^{1,2}$}

'Center for Laboratory Medicine, ${ }^{2}$ Center for Hemostaseology and Hemophilia, St Gallen, Switzerland
Correspondence: Wolfgang Korte Center for Laboratory Medicine, Frohbergstrasse 3, St Gallen 9007, Switzerland

Tel +4I 7| 4943902

Fax +4I 7| 4943900

Email wolfgang.korte@zlmsg.ch
Abstract: Circulating factor XIII (FXIII) consists of two active (A) and two carrier (B) subunits in tetrameric form. Congenital FXIII deficiency is a rare autosomal-recessive trait that mostly results from an FXIII A-subunit deficiency. Classic coagulation assays, such as prothrombin time or activated partial thromboplastin time, are not sensitive to FXIII; therefore, specific FXIII assays are necessary to detect the deficiency. The clinical picture of congenital FXIII deficiency comprises abortions, umbilical cord bleeding, increased surgical bleeding, intracerebral hemorrhage (which can, unfortunately, be the very first sign of severe FXIII deficiency), menorrhagia, and wound-healing disorders. Given the risk of intracranial hemorrhage, continued prophylaxis is to be recommended in severe deficiency, even in the actual absence of bleeding symptoms. Functional FXIII half-life decreases in consumptive processes (eg, surgery), explaining why increased dosing is needed in such situations. A recombinant FXIII (rFXIII) subunit-A molecule, which is expressed in Saccharomyces cerevisiae, has been evaluated for replacement therapy in congenital FXIII deficiency. The bleeding frequency under continued rFXIII prophylaxis during a year-long treatment period was significantly lower compared to on-demand treatment. Importantly, no severe spontaneous bleedings occurred, and bleeding requiring additional intervention only occurred after relevant trauma. Treatment with rFXIII proved to be safe: antibodies against rFXIII detected in four patients were not considered clinically relevant. No allergic reactions were observed. These data show that rFXIII can be used safely and effectively for continued prophylaxis in congenital FXIII deficiency; it is conceivable that this also holds true for treatment of acute bleeding, but clinical proof of this is pending.

Keywords: FXIII, transglutaminase, bleeding, clot firmness

\section{Introduction}

Hemostasis represents a tightly regulated system of various pathways to ensure an optimal equilibrium between excessive bleeding and thrombosis. A clot formed in the presence of calcium ions is usually resistant to proteolytic breakdown, suggesting the presence of an "insoluble" clot. It was noticed early on by Lorand ${ }^{1}$ that a single factor (initially called "fibrin-stabilizing factor") is the main one responsible for the mechanical characteristics of insoluble fibrin clots. In 1960, Duckert et al described the first case of a previously unknown congenital hemorrhagic diathesis, which was due to the deficiency of this fibrin-stabilizing factor, or factor XIII (FXIII); ${ }^{2}$ the main biochemical abnormality observed at the time was the solubility of the clot in $5 \mathrm{M}$ urea, and it was not until recently that this case was genetically characterized. ${ }^{3}$

Congenital FXIII deficiency is an autosomal-recessive disease, ${ }^{4}$ with an estimated prevalence of approximately one in four million worldwide, but with likely clustering. 
Only a few more than 300 cases have been reported, predominantly in Japan. ${ }^{5}$ Congenital FXIII deficiency seems, expectedly, more frequent in families with consanguineous marriages, eg, in South Asia. ${ }^{6}$ In Pakistan, $80 \%$ of the cases detected in a case series over a 10 -year period occurred in families with consanguineous marriages. ${ }^{?}$

The clinical significance of FXIII in the maintenance of adequate hemostasis can be deducted from hemorrhagic symptoms observed in patients with FXIII deficiency or inhibiting FXIII antibodies. ${ }^{8,9}$ The clinical importance of acquired FXIII deficiency with consumptive processes has been recognized more recently. ${ }^{10,11}$ Besides hemostasis, FXIII seems also to play important roles in phagocytosis ${ }^{12}$ and tissue repair. ${ }^{13}$ Furthermore, FXIII is important in maintaining pregnancies, ${ }^{14,15}$ as deficiencies are associated with a higher rate of miscarriages. ${ }^{16}$ FXIII deficiency has also been shown to be associated with poor wound healing ${ }^{17-19}$ and angiogenesis. ${ }^{20,21}$ Other important functions of FXIII comprise osteoblast function ${ }^{22,23}$ and the support of the innate immune response to bacteria. ${ }^{24}$

Polymorphisms of the FXIII gene lead to changes in clot properties ${ }^{25}$ that seem to be clinically relevant; while intracranial hemorrhage seems more prevalent in patients with the FXIII Val34Leu polymorphism,${ }^{26}$ less myocardial infarctions seem to occur. ${ }^{27,28}$ This exemplifies that FXIII is apparently situated at the interface between the maintenance of clot integrity and clot breakdown. ${ }^{29}$

\section{Structure-function relationship}

Circulating FXIII consists of two identical proenzyme $\left(\mathrm{A}_{2}\right)$ and carrier-protein $\left(\mathrm{B}_{2}\right)$ subunits. The A subunit contains the active center, the activation peptide, a calcium-binding site, and free sulfhydryl groups. The B subunit acts as a carrier protein to stabilize subunit $\mathrm{A}$; it also binds fibrinogen and assists in regulating FXIII activity. ${ }^{30,31}$ The $\mathrm{B}$ subunit is found in plasma in its free form as well as a part of the $\mathrm{A}_{2} \mathrm{~B}_{2}$ tetramer. Usually, the A subunit is completely complexed with B subunits; therefore, the A subunit in its inactive state is found in the tetramer only. Factor XIII can be found circulating in association with fibrinogen. ${ }^{32}$

The gene for subunit A is on chromosome 6 (p24-p25), consists of 160 kilobases and has 15 exons. Mutations or defects associated with FXIII deficiency seem to be without a cluster, but are spread out over the FXIII-A gene, and the mutations seem to be associated with the severity of the disease. ${ }^{33-35}$ The mutations (more than 100 described) are mostly missense and nonsense mutations; the gene for the $\mathrm{B}$ subunit does not seem to be involved in these cases. The subunit-B gene, consisting of 28 kilobases and with 12 exons, is located on chromosome 1 (q31-q32.1). ${ }^{36-38}$ Very few mutations that lead to FXIII-B deficiency have been described. ${ }^{39}$

A very frequent FXIII polymorphism (Val34Leu) seems to play no part in severe (congenital) FXIII deficiency but contributes to the wide range of ("normal") distribution of FXIII activity. ${ }^{40}$ Synthesis of subunit A occurs in bone marrow cells, monocytes, and macrophages, as well as hepatocytes; subunit B is synthesized in hepatocytes. ${ }^{41,42}$ About $50 \%$ of the total FXIII activity is present in platelets as $\mathrm{A}_{2}$ homodimers. This "tissue transglutaminase 2" - being in fact the intracellular form of FXIII - is found as $\mathrm{A}_{2}$ homodimer in platelets, megakaryocytes, monocytes, macrophages, the liver, placenta, and uterus, ${ }^{36,42-46}$ whereas a clinically relevant contribution of monocytes seems questionable. ${ }^{47}$ During steady-state situations, FXIII has a long functional half-life of approximately 10 days, ${ }^{48}$ but it is likely to be shorter during consumption (eg, bleeding) situations ${ }^{49}$ or with subunit-B deficiency. ${ }^{50}$

Upon thrombin generation, fibrinopeptide $A$ is released from the fibrinogen $\alpha$-chain and fibrinopeptide B from the fibrinogen $\beta$-chain. These steps mark the transition to the fibrin monomer molecule. FXIIIa is generated after thrombinmediated cleavage of an activation peptide $;{ }^{51}$ fibrin itself is a cofactor in the activation of FXIII to FXIIIa, accelerating the activation process. The subsequent calcium-dependent dissociation of the B subunit allows the presentation of the active center through conformational changes. ${ }^{52-54}$ Fibrin monomers associate spontaneously in a longitudinal and lateral fashion to form (soluble) fibrin strands. Cross-linking by FXIIIa then generates a mechanically stable, insoluble fibrin clot by cross-linking fibrinogen glutamyl and lysine residues through isopeptide bonds.

Apart from its clot-stabilizing properties, FXIII also anchors $\alpha 2$-antiplasmin to the fibrin clot as well as fibrinogen. ${ }^{55,56}$ That way, FXIII activity not only ensures crosslinking and thus mechanical stability of the clot, but at the same time it also guarantees a certain fibrinolytic resistance of the clot and the substrate used to build a fibrin clot.

\section{Detecting and categorizing FXIII deficiencies}

FXIII deficiency does not influence classic global coagulation tests, such as prothrombin time (PT)/international normalized ratio (INR) or activated partial thromboplastin time (aPTT), to a clinically relevant extent. Therefore, normal INR or aPTT values do not allow the exclusion of FXIII deficiency, and specific methods need to be employed. At present, most 
laboratories use a chromogenic assay, which determines the effect of subunit-A activity. ${ }^{57}$ This assay seems to be less accurate in the low range when compared to an incorporation assay based on the natural function of FXIII, ${ }^{58}$ which is a modification of earlier assays..$^{59,60}$ Antigen measurements have for a long time been associated with increased resource use and longer turnaround times, making them most relevant for scientific research and less so for patient management. The determination of the $\mathrm{A}_{2} \mathrm{~B}_{2}$ tetramer is recommended before such further steps as specific subunit determination or mixing assays (if an inhibitor has to be taken into consideration) are undertaken. ${ }^{18}$ However, in daily clinical practice, the detection of FXIII (ie, subunit A) activity is most important, and will correlate in the vast majority of the cases with subunit-A concentration. Immunoassays to detect the FXIII-A subunit on autoanalyzers have recently been marketed.

As FXIII measurements become more and more important in acute-care bleeding management, development of assays that allow a very short turnaround time would be of advantage. It was shown that FXIII deficiency significantly influences whole-blood thrombelastographic assays, and proof-of-principle studies suggest that whole-blood aggregometry can be used for the fast and reliable determination of a potential FXIII deficiency. ${ }^{61}$

As mentioned, patients with congenital FXIII deficiency mostly show aberrations in the FXIII-A gene. ${ }^{34,35,62}$ While this leads to direct impairment of FXIII activity through either qualitative or quantitative defects of the A subunit, mutations in the FXIII-B gene lead to decreased FXIII-B expression, and thus in turn to increased FXIII-A degradation (of the normal FXIII-A subunit), due to missing protection by the FXIII-B subunit. ${ }^{50}$ Therefore, congenital FXIII deficiency is predominately divided into FXIII-A and FXIII-B deficiency, whereas FXIII-A deficiency can theoretically be further subclassified into quantitative (type 1) and qualitative (type 2) defects.

Acquired FXIII deficiency can be frequently detected in patients with preexisting diseases and ongoing, albeit subclinical, coagulation activation, suggesting that consumption is the main origin of these deficiencies. If these patients undergo surgical stress, bleeding symptoms can occur, and FXIII replacement seems beneficial in these cases. In our experience, acquired FXIII deficiency is frequent, and most of the time not related to inhibitors or drug use, but to the perioperative setting. ${ }^{11,63,64}$

\section{The clinical picture}

Congenital FXIII deficiency can manifest very early on in life as bleeding from the umbilical stump. ${ }^{65}$ Registry data have shown that intracranial hemorrhage (which can, unfortunately, be the very first sign of severe FXIII deficiency; this is often preceded by trauma in children; intracerebral bleeding may recur in up to a third of cases with a correspondingly high risk of mortality), umbilical cord bleeding (up to 3 weeks after birth), and intramuscular and intracutaneous bleeding are the most frequent bleeding types in congenital FXIII deficiency, with intracranial hemorrhage and umbilical stump bleeding being much more frequent in FXIII deficiency than other coagulation-factor deficiencies. ${ }^{66}$ Umbilical bleeding due to FXIII deficiency might be life-threatening in neonates, which is why aggressive diagnostics and replacement therapy are necessary once FXIII deficiency is considered or recognized. Other clinical signs associated with FXIII deficiency $6,7,17,67-70$ include spontaneous abortions in early pregnancy; a general bleeding tendency, especially with trauma (this bleeding may be immediate, delayed or repeated); menorrhagia; and wound-healing disorders, which are a common finding.

Peri- and postoperative bleeding is another frequent bleeding type in congenital FXIII deficiency; however, acquired FXIII deficiency is a frequent finding in the perioperative setting, and might contribute - alone or in combination with a preexisting congenital deficiency - to a bleeding diathesis in this setting. ${ }^{10,11,63,64}$ In patients with congenital FXIII deficiency, it seems that low levels of FXIII (in the single-figure percentage range) are sufficient to provide clinically adequate hemostasis ${ }^{71}$ in a steady-state setting. Retrospective as well as prospective data suggest that we need to differentiate between a congenital and a congenital-plus-acquired deficiency with regard to clinical management. In the acquired-deficiency setting, clinical symptoms seem much more pronounced (ie, occurring at much-higher factor levels) compared to the congenital deficiency setting. ${ }^{10,11,72-75}$

\section{Treatment options for congenital FXIII deficiency}

Congenital, symptomatic FXIII deficiency is overall an infrequent finding; as mentioned earlier, the clinical picture is somewhat variable, depending on the severity of the deficiency. ${ }^{66,76}$ Long-term replacement therapy for (severe) congenital FXIII deficiency needs always to be considered because of the high risk of central nervous system hemorrhage. As the half-life of FXIII is long in a steady-state situation, replacement is often only needed every 4 weeks, ${ }^{77}$ but the frequency of replacement should be adapted to the clinical need; in steady-state situations, 10-20 IU should be given per kilogram of body weight every 4 weeks. 
Theoretically, such doses can be given through the application of fresh frozen plasma; however, the volume needed (approximately $20 \mathrm{~mL} / \mathrm{kg}$ ) and the logistics of a frozen material compared to a lyophilized substance make lyophilized factor concentrates the ideal solution for replacement therapy. Various studies showed prophylactic treatment with a plasma-derived product to be effective without relevant toxicity. ${ }^{78-80}$ Similar observations with a stringent evaluation protocol have recently been made for recombinant FXIII (catridecacog) in volunteers, ${ }^{81}$ as well as patients. ${ }^{26,82,83}$ Patients with FXIII below 1\% should receive immediate prophylaxis, even if no bleeding complications have occurred. ${ }^{80}$ For patients with levels of $1 \%-4 \%$, primary prophylaxis should also be considered, as there is still a high chance of severe bleeding complications. With FXIII levels $>5 \%$, it is generally assumed that no spontaneous severe bleeding will occur, given that FXIII levels of 3\%-10\% of the normal population mean are sufficient to prevent spontaneous bleeds. ${ }^{84}$ However, bleeding might still occur ${ }^{65}$ and might be significant (eg, if surgical stress is applied); therefore, higher-than-prophylactic levels are recommended in FXIIIdeficient patients undergoing surgery. ${ }^{63,85}$ In pregnancy, more frequent replacements with lower doses have been reported to be successful, ${ }^{69}$ which is important, as pregnant women with severe congenital FXIII deficiency have a roughly $50 \%$ chance of experiencing recurrent pregnancy losses or spontaneous abortions. ${ }^{86,87}$ Special caution needs to be taken to secure adequate plasma levels during labor in order to prevent hemorrhagic complications. ${ }^{69}$

In acute bleeding episodes, FXIII replacement should be sufficiently dosed (eg, at $30 \mathrm{IU} / \mathrm{kg})^{10}$ to reach $60 \%$ activity levels, and with severe or intracranial bleeding, continued monitoring of FXIII levels is mandatory to guarantee sufficient replacement (as half-life is dramatically shortened with situations where consumption is going on). Unlike the purified plasma product, which is essentially the purified $\mathrm{A}_{2} \mathrm{~B}_{2}$ tetramer, the recently introduced recombinant FXIII- $\mathrm{A}_{2}$ (rFXIII-A ${ }_{2}$, catridecacog) ${ }^{26}$ combines with free endogenous FXIII-B in plasma to form a functional tetramer. Therefore, patients with a congenital deficiency of the B unit can be expected to have a shortened functional half-life after application of the rFXIII; it is conceivable that rFXIII-A $\mathrm{A}_{2}$ will be functional in B-subunit deficiency, but this awaits clinical confirmation, and until then it remains an off-label indication. Catridecacog was shown to be effective and safe in patients with congenital FXIII deficiency undergoing prophylactic replacement therapy. ${ }^{26,82,83}$ While low-titer antibodies against the recombinant protein were detected in some patients, there was no evidence that they were of any clinical relevance. ${ }^{82}$ Both the efficacy and the safety profile in pediatric patients were also found to be good. ${ }^{83}$ Mean half-life of the new drug under steady-state conditions was found to be approximately 9-15 days. ${ }^{26,83}$ In surgical patients, the use of recombinant FXIII (catridecacog) on top of normal FXIII values was not associated with any safety issues, providing additional proof of the safety of such a treatment approach. ${ }^{88}$

\section{Conclusion}

Congenital FXIII deficiency is rare, with a prevalence of approximately one in four million. This autosomal-recessive disorder appears to be underdiagnosed, as it is not recognized by classic global tests, such as aPTT or PT. Specific assays need to be implemented and performed to detect the deficiency; early recognition is essential to allow adequate prophylactic therapy, which might save patients from life-threatening bleeding and the potentially related morbidity and mortality. Currently, standard therapy for prophylaxis is performed using plasmatic FXIII concentrate. However, recombinant FXIII (catridecacog) is now commercially available, and has proven to be safe and effective in bleeding prophylaxis with congenital FXIII deficiency. Replacing plasmatic concentrates with recombinant products will render this replacement therapy independent of the need for blood donations. As acquired FXIII deficiency with bleeding complications is very frequent in the peri-interventional setting, further studies will need to evaluate whether the recombinant product can replace the plasmatic concentrate in the noncongenital deficiency setting too. This will ultimately decide whether catridecacog is a breakthrough in FXIII-replacement therapy.

\section{Disclosure}

The author has received speaker fees, consulting fees, and travel support from Novo Nordisk, CSL Behring, Baxter, DSM Pentapharm, Axonlab, and Roche Diagnostics.

\section{References}

1. Lorand L. Fibrinoligase: the fibrin-stabilizing factor system of blood plasma. Ann N Y Acad Sci. 1972;202:6-30.

2. Duckert F, Jung E, Shmerling DH. A hitherto undescribed congenital haemorrhagic diathesis probably due to fibrin stabilizing factor deficiency. Thromb Diath Haemorrh. 1960;5:179-186.

3. Schroeder V, Meili E, Cung T, Schmutz P, Kohler HP. Characterisation of six novel A-subunit mutations leading to congenital factor XIII deficiency and molecular analysis of the first diagnosed patient with this rare bleeding disorder. Thromb Haemost. 2006;95(1):77-84.

4. Board PG, Losowsky MS, Miloszewski KJ. Factor XIII: inherited and acquired deficiency. Blood Rev. 1993;7(4):229-242.

5. Fadoo Z, Merchant Q, Rehman KA. New developments in the management of congenital factor XIII deficiency. J Blood Med. 2013;4: $65-73$. 
6. Bhattacharya M, Biswas A, Ahmed RP, et al. Clinico-hematologic profile of factor XIII-deficient patients. Clin Appl Thromb Hemost. 2005;11(4):475-480.

7. Fadoo Z, Saleem AF. Factor XIII deficiency in children - clinical presentation and outcome. J Coll Physicians Surg Pak. 2008;18(9): 565-568.

8. Nakamura S, Kato A, Sakata Y, Aoki N. Bleeding tendency caused by IgG inhibitor to factor XIII, treated successfully by cyclophosphamide. Br J Haematol. 1988;68(3):313-319.

9. Lorand L, Velasco PT, Rinne JR, Amare M, Miller LK, Zucker ML. Autoimmune antibody (IgG Kansas) against the fibrin stabilizing factor (factor XIII) system. Proc Natl Acad Sci U S A. 1988;85(1):232-236.

10. Korte WC, Szadkowski C, Gahler A, et al. Factor XIII substitution in surgical cancer patients at high risk for intraoperative bleeding. Anesthesiology. 2009;110(2):239-245.

11. Wettstein P, Haeberli A, Stutz M, et al. Decreased factor XIII availability for thrombin and early loss of clot firmness in patients with unexplained intraoperative bleeding. Anesth Analg. 2004;99(5):1564-1569.

12. Sárváry A, Szucs S, Balogh I, et al. Possible role of factor XIII subunit A in Fcgamma and complement receptor-mediated phagocytosis. Cell Immunol. 2004;228(2):81-90.

13. Nahrendorf M, Sosnovik DE, Waterman P, et al. Dual channel optical tomographic imaging of leukocyte recruitment and protease activity in the healing myocardial infarct. Circ Res. 2007;100(8):1218-1225.

14. Koseki-Kuno S, Yamakawa M, Dickneite G, Ichinose A. Factor XIII A subunit-deficient mice developed severe uterine bleeding events and subsequent spontaneous miscarriages. Blood. 2003;102(13): 4410-4412.

15. Parameswaran KN, Cheng XF, Chen EC, Velasco PT, Wilson JH, Lorand L. Hydrolysis of gamma:epsilon isopeptides by cytosolic transglutaminases and by coagulation factor XIIIa. J Biol Chem. 1997;272(15):10311-10317.

16. Kappelmayer J, Bacsko G, Kelemen E, Adany R. Onset and distribution of factor XIII-containing cells in the mesenchyme of chorionic villi during early phase of human placentation. Placenta. 1994;15(6):613-623.

17. Inbal A, Lubetsky A, Krapp T, et al. Impaired wound healing in factor XIII deficient mice. Thromb Haemost. 2005;94(2):432-437.

18. Muszbek L, Bagoly Z, Cairo A, Peyvandi F. Novel aspects of factor XIII deficiency. Curr Opin Hematol. 2011;18(5):366-372.

19. Becker SW, Weidt F, Rohl K. The role of plasma transglutaminase (F XIII) in wound healing of complicated pressure sores after spinal cord injury. Spinal Cord. 2001;39(2):114-117.

20. Inbal A, Dardik R. Role of coagulation factor XIII (FXIII) in angiogenesis and tissue repair. Pathophysiol Haemost Thromb. 2006;35(1-2): $162-165$.

21. Chung SI, Lee SY, Ryogin U, Kamemitsu K. Affects [sic] of F XIII in wound-healing - fibrin stability in tissues and cross linking of angiogenesis modulator, osteonectin to fibrin. Rinsho Byori. 1997; Suppl 104:50.

22. Piercy-Kotb SA, Mousa A, Al-Jallad HF, et al. Factor XIIIA transglutaminase expression and secretion by osteoblasts is regulated by extracellular matrix collagen and the MAP kinase signaling pathway. J Cell Physiol. 2012;227(7):2936-2946.

23. Al-Jallad HF, Myneni VD, Piercy-Kotb SA, et al. Plasma membrane factor XIIIA transglutaminase activity regulates osteoblast matrix secretion and deposition by affecting microtubule dynamics. PLoS One. 2011;6(1):e15893.

24. Loof TG, Morgelin M, Johansson L, et al. Coagulation, an ancestral serine protease cascade, exerts a novel function in early immune defense. Blood. 2011;118(9):2589-2598.

25. Ariens RA, Philippou H, Nagaswami C, Weisel JW, Lane DA, Grant PJ. The factor XIII V34L polymorphism accelerates thrombin activation of factor XIII and affects cross-linked fibrin structure. Blood. 2000;96(3):988-995.

26. Lovejoy AE, Reynolds TC, Visich JE, et al. Safety and pharmacokinetics of recombinant factor XIII-A2 administration in patients with congenital factor XIII deficiency. Blood. 2006;108(1):57-62.
27. Rallidis LS, Politou M, Komporozos C, et al. Factor XIII Val34Leu polymorphism and the risk of myocardial infarction under the age of 36 years. Thromb Haemost. 2008;99(6):1085-1089.

28. Shafey M, Anderson JL, Scarvelis D, Doucette SP, Gagnon F, Wells PS Factor XIII Val34Leu variant and the risk of myocardial infarction: a meta-analysis. Thromb Haemost. 2007;97(4):635-641.

29. Loewy AG. Some thoughts on the state in nature, biosynthetic origin, and function of factor XIII. Ann N Y Acad Sci. 1972;202:41-58.

30. Siebenlist KR, Meh DA, Mosesson MW. Plasma factor XIII binds specifically to fibrinogen molecules containing gamma chains. Biochemistry. 1996;35(32):10448-10453.

31. Siebenlist KR, Meh DA, Mosesson MW. Protransglutaminase (factor XIII) mediated crosslinking of fibrinogen and fibrin. Thromb Haemost. 2001;86(5):1221-1228.

32. Mosesson MW, Siebenlist KR, Meh DA. The structure and biological features of fibrinogen and fibrin. Ann NY Acad Sci. 2001;936:11-30.

33. Andersen MD, Kjalke M, Bang S, et al. Coagulation factor XIII variants with altered thrombin activation rates. Biol Chem. 2009;390(12): 1279-1283.

34. Anwar R, Valleley EM, Trinh CH. Novel human pathological mutations. Gene symbol: F13A1. Disease: factor xiii deficiency. Hum Genet. 2010;127(1):115-116.

35. Anwar R, Gallivan L, Richards M, Khair K, Wright M, Minford A. Factor XIII deficiency: new nonsense and deletion mutations in the human factor XIIIA gene. Haematologica. 2005;90(12): $1718-1720$.

36. Weisberg LJ, Shiu DT, Greenberg CS, Kan YW, Shuman MA. Localization of the gene for coagulation factor XIII a-chain to chromosome 6 and identification of sites of synthesis. J Clin Invest. 1987;79(2): 649-652.

37. Ichinose A, Hendrickson LE, Fujikawa K, Davie EW. Amino acid sequence of the a subunit of human factor XIII. Biochemistry. 1986;25(22):6900-6906.

38. Ichinose A, McMullen BA, Fujikawa K, Davie EW. Amino acid sequence of the $\mathrm{b}$ subunit of human factor XIII, a protein composed of ten repetitive segments. Biochemistry. 1986;25(16):4633-4638.

39. Koseki S, Souri M, Koga S, et al. Truncated mutant B subunit for factor XIII causes its deficiency due to impaired intracellular transportation. Blood. 2001;97(9):2667-2672.

40. Kangsadalampai S, Board PG. The Val34Leu polymorphism in the A subunit of coagulation factor XIII contributes to the large normal range in activity and demonstrates that the activation peptide plays a role in catalytic activity. Blood. 1998;92(8):2766-2770.

41. Nagy JA, Henriksson P, McDonagh J. Biosynthesis of factor XIII B subunit by human hepatoma cell lines. Blood. 1986;68(6):1272-1279.

42. Kaczmarek E, Liu Y, Berse B, Chen CS, McDonagh J. Biosynthesis of plasma factor XIII: evidence for transcription and translation in hepatoma cells. Biochim Biophys Acta. 1995;1247(1):127-134.

43. Muszbek L, Adany R, Mikkola H. Novel aspects of blood coagulation factor XIII. I. Structure, distribution, activation, and function. Crit Rev Clin Lab Sci. 1996;33(5):357-421.

44. Adany R, Muszbek L. Immunohistochemical detection of factor XIII subunit a in histiocytes of human uterus. Histochemistry. 1989;91(2): $169-174$.

45. Fear JD, Jackson P, Gray C, Miloszewski KJ, Losowsky MS. Localisation of factor XIII in human tissues using an immunoperoxidase technique. J Clin Pathol. 1984;37(5):560-563.

46. Weisberg LJ, Shiu DT, Conkling PR, Shuman MA. Identification of normal human peripheral blood monocytes and liver as sites of synthesis of coagulation factor XIII a-chain. Blood. 1987;70(2):579-582.

47. Inbal A, Muszbek L, Lubetsky A, et al. Platelets but not monocytes contribute to the plasma levels of factor XIII subunit A in patients undergoing autologous peripheral blood stem cell transplantation. Blood Coagul Fibrinolysis. 2004;15(3):249-253.

48. Fear JD, Miloszewski KJ, Losowsky MS. The half life of factor XIII in the management of inherited deficiency. Thromb Haemost. 1983;49(2):102-105. 
49. Zimmermann R, Ehlers W, Manz F, Meinrenken W, Egbring R, Gemmeke H. [Severe factor XIII-deficiency. Studies on subunits and turnover of the fibrin stabilizing factor (author's trans1)]. Blut. 1977;35(6):457-464. German.

50. Saito M, Asakura H, Yoshida T, et al. A familial factor XIII subunit B deficiency. Br J Haematol. 1990;74(3):290-294.

51. Schroeder V, Vuissoz JM, Caflisch A, Kohler HP. Factor XIII activation peptide is released into plasma upon cleavage by thrombin and shows a different structure compared to its bound form. Thromb Haemost. 2007;97(6):890-898.

52. Mosesson MW. Fibrinogen and fibrin polymerization: appraisal of the binding events that accompany fibrin generation and fibrin clot assembly. Blood Coagul Fibrinolysis. 1997;8(5):257-267.

53. Casadio R, Polverini E, Mariani P, et al. The structural basis for the regulation of tissue transglutaminase by calcium ions. Eur J Biochem. 1999;262(3):672-679.

54. Fox BA, Yee VC, Pedersen LC, et al. Identification of the calcium binding site and a novel ytterbium site in blood coagulation factor XIII by X-ray crystallography. J Biol Chem. 1999;274(8):4917-4923.

55. Tamaki T, Aoki N. Cross-linking of alpha 2-plasmin inhibitor to fibrin catalyzed by activated fibrin-stabilizing factor. $J$ Biol Chem. 1982;257(24):14767-14772.

56. Mosesson MW, Siebenlist KR, Hernandez I, Lee KN, Christiansen VJ, McKee PA. Evidence that alpha2-antiplasmin becomes covalently ligated to plasma fibrinogen in the circulation: a new role for plasma factor XIII in fibrinolysis regulation. J Thromb Haemost. 2008;6(9): $1565-1570$.

57. Fickenscher K, Aab A, Stüber W. A photometric assay for blood coagulation factor XIII. Thromb Haemost. 1991;65(5):535-540.

58. Wilmer M, Rudin K, Kolde H, et al. Evaluation of a sensitive colorimetric FXIII incorporation assay. effects of FXIII val34leu, plasma fibrinogen concentration and congenital FXIII deficiency. Thromb Res. 2001;102(1):81-91.

59. Dempfle CE, Harenberg J, Hochreuter K, Heene DL. Microtiter assay for measurement of factor XIII activity in plasma. J Lab Clin Med. 1992;119(5):522-528.

60. Nishida Y, Ikematsu S, Fukutake K, Fujimaki M, Fukutake K, Kakishita E. A new rapid and simple assay for factor XIII activity using dansylcadaverine incorporation and gel filtration. Thromb Res. 1984;36(2):123-131.

61. Jambor C, Reul V, Schnider TW, Degiacomi P, Metzner H, Korte WC. In vitro inhibition of factor XIII retards clot formation, reduces clot firmness, and increases fibrinolytic effects in whole blood. Anesth Analg. 2009;109(4):1023-1028.

62. Muszbek L. Deficiency causing mutations and common polymorphisms in the factor XIII-A gene. Thromb Haemost. 2000;84(4):524-527.

63. Haas T, Korte W, Spielmann N, et al. Perioperative course of FXIII in children undergoing major surgery. Paediatr Anaesth. 2012;22(7): 641-646.

64. Korte W. F XIII in perioperative coagulation management. Best Pract Res Clin Anaesthesiol. 2010;24(1):85-93.

65. Anwar R, Minford A, Gallivan L, Trinh CH, Markham AF. Delayed umbilical bleeding - a presenting feature for factor XIII deficiency: clinical features, genetics, and management. Pediatrics. 2002;109(2):E32

66. Ivaskevicius V, Seitz R, Kohler HP, et al. International registry on factor XIII deficiency: a basis formed mostly on European data. Thromb Haemost. 2007;97(6):914-921.

67. Schroeder V, Durrer D, Meili E, Schubiger G, Kohler HP. Congenital factor XIII deficiency in Switzerland: from the worldwide first case in 1960 to its molecular characterisation in 2005. Swiss Med Wkly. 2007;137(19-20):272-278.

68. Padmanabhan LD, Mhaskar R, Mhaskar A, Ross CR. Factor XIII deficiency: a rare cause of repeated abortions. Singapore Med J. 2004;45(4):186-187.
69. Asahina T, Kobayashi T, Takeuchi K, Kanayama N. Congenital blood coagulation factor XIII deficiency and successful deliveries: a review of the literature. Obstet Gynecol Surv. 2007;62(4):255-260.

70. Egbring R, Kröniger A, Seitz R. Factor XIII deficiency: pathogenic mechanisms and clinical significance. Semin Thromb Hemost. 1996;22(5):419-425.

71. Kitchens CS, Newcomb TF. Factor XIII. Medicine (Baltimore). 1979;58(6):413-429.

72. Gerlach R, Raabe A, Zimmermann M, Siegemund A, Seifert V. Factor XIII deficiency and postoperative hemorrhage after neurosurgical procedures. Surg Neurol. 2000;54(3):260-264; discussion 264-265.

73. Gerlach R, Tölle F, Raabe A, Zimmermann M, Siegemund A, Seifert V. Increased risk for postoperative hemorrhage after intracranial surgery in patients with decreased factor XIII activity: implications of a prospective study. Stroke. 2002;33(6):1618-1623.

74. Gödje O, Haushofer M, Lamm P, Reichart B. The effect of factor XIII on bleeding in coronary surgery. Thorac Cardiovasc Surg. 1998;46(5): 263-267.

75. Gödje O, Gallmeier U, Schelian M, Grünewald M, Mair H. Coagulation factor XIII reduces postoperative bleeding after coronary surgery with extracorporeal circulation. Thorac Cardiovasc Surg. 2006;54(1): 26-33.

76. Ivaskevicius V, Windyga J, Baran B, et al. Phenotype-genotype correlation in eight Polish patients with inherited factor XIII deficiency: identification of three novel mutations. Haemophilia. 2007;13(5): 649-657.

77. Meili EO. [Clinical course and management of severe congenital factor XIII deficiency]. Hamostaseologie. 2002;22(1):48-52. German.

78. Nugent D. Corifact/Fibrogammin P in the prophylactic treatment of hereditary factor XIII deficiency: results of a prospective, multicenter, open-label study. Thromb Res. 2012;130 Suppl 2:S12-S14.

79. Gootenberg JE. Factor concentrates for the treatment of factor XIII deficiency. Curr Opin Hematol. 1998;5(6):372-375.

80. Lusher J, Pipe SW, Alexander S, Nugent D. Prophylactic therapy with Fibrogammin $\mathrm{P}$ is associated with a decreased incidence of bleeding episodes: a retrospective study. Haemophilia. 2010;16(2): 316-321.

81. Visich JE, Zuckerman LA, Butine MD, et al. Safety and pharmacokinetics of recombinant factor XIII in healthy volunteers: a randomized, placebo-controlled, double-blind, multi-dose study. Thromb Haemost. 2005;94(4):802-807.

82. Inbal A, Oldenburg J, Carcao M, Rosholm A, Tehranchi R, Nugent D. Recombinant factor XIII: a safe and novel treatment for congenital factor XIII deficiency. Blood. 2012;119(22):5111-5117.

83. Williams M, Will A, Stenmo C, Rosholm A, Tehranchi R. Pharmacokinetics of recombinant factor XIII in young children with congenital FXIII deficiency and comparison with older patients. Haemophilia. 2014;20(1):99-105.

84. Castaman G. Prophylaxis of bleeding episodes and surgical interventions in patients with rare inherited coagulation disorders. Blood Transfus. 2008;6 Suppl 2:s39-s44.

85. Idris Z, Muzaimi M, Hussin S, Mahmood WH, Abdullah WZ. Association of perioperative factor XIII activity levels and other haemostatic markers with the risk of postoperative intracranial haematoma in a selected cohort of neurosurgical patients. Acta Neurochir (Wien). 2012;154(5):887-893; discussion 893-894.

86. Ichinose A, Asahina T, Kobayashi T. Congenital blood coagulation factor XIII deficiency and perinatal management. Curr Drug Targets. 2005;6(5):541-549.

87. Inbal A, Muszbek L. Coagulation factor deficiencies and pregnancy loss. Semin Thromb Hemost. 2003;29(2):171-174.

88. Karkouti K, von Heymann C, Jespersen CM, et al. Efficacy and safety of recombinant factor XIII on reducing blood transfusions in cardiac surgery: a randomized, placebo-controlled, multicenter clinical trial. J Thorac Cardiovasc Surg. 2013;146(4):927-939. 
Journal of Blood Medicine

\section{Publish your work in this journal}

The Journal of Blood Medicine is an international, peer-reviewed, open access, online journal publishing laboratory, experimental and clinical aspects of all topics pertaining to blood based medicine including but not limited to: Transfusion Medicine; Blood collection, Donor issues, Transmittable diseases, and Blood banking logistics; Immunohematology; Artificial and alternative

blood based therapeutics; Hematology; Biotechnology/nanotechnology of blood related medicine; Legal aspects of blood medicine; Historical perspectives. The manuscript management system is completely online and includes a very quick and fair peer-review system. Visit http://www.dovepress.com/ testimonials.php to read real quotes from published authors.

Submit your manuscript here: http://www.dovepress.com/Journal-of-blood-medicine-journal 